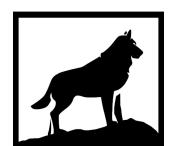

Michigan Technological

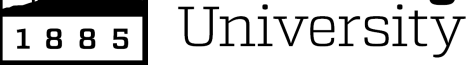

Michigan Technological University Digital Commons @ Michigan Tech

DEFECT-DEFERRED CORRECTION METHOD FOR THE TWODOMAIN CONVECTION-DOMINATED CONVECTION-DIFFUSION PROBLEM

Dilek Erkmen

Michigan Technological University, derkmen@mtu.edu

Copyright 2016 Dilek Erkmen

Recommended Citation

Erkmen, Dilek, "DEFECT-DEFERRED CORRECTION METHOD FOR THE TWO-DOMAIN CONVECTIONDOMINATED CONVECTION-DIFFUSION PROBLEM", Open Access Master's Thesis, Michigan Technological University, 2016.

https://doi.org/10.37099/mtu.dc.etdr/160

Follow this and additional works at: https://digitalcommons.mtu.edu/etdr

Part of the Numerical Analysis and Computation Commons, and the Partial Differential Equations Commons 


\title{
DEFECT-DEFERRED CORRECTION METHOD FOR THE TWO-DOMAIN CONVECTION-DOMINATED CONVECTION-DIFFUSION PROBLEM
}

By

Dilek Erkmen

\begin{abstract}
A THESIS
Submitted in partial fulfillment of the requirements for the degree of MASTER OF SCIENCE

In Mathematical Sciences
\end{abstract}

MICHIGAN TECHNOLOGICAL UNIVERSITY

Copr. 2016 Dilek Erkmen 
This thesis has been approved in partial fulfillment of the requirements for the Degree of MASTER OF SCIENCE in Mathematical Sciences.

Department of Mathematical Sciences

Thesis Advisor : Alexander Labovsky

Committee Member : Allan Struthers

Committee Member : Aleksandr Sergeyev

Department Chair : Mark S. Gockenbach 


\section{Contents}

1 List of Tables $\ldots \ldots \ldots \ldots \ldots \ldots \ldots \ldots \ldots \ldots \ldots \ldots$ iv

2 Acknowledgements $\ldots \ldots \ldots \ldots \ldots \ldots \ldots \ldots \ldots \ldots \ldots \ldots$

$3 \quad$ Abstract........................... vi

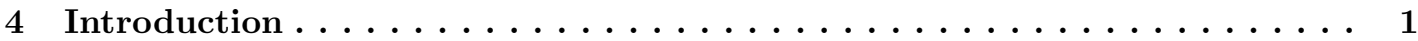

5 Method Description, Notation and Preliminaries............. 5

5.1 Discrete Formulation . . . . . . . . . . . . . . . . . . . . 6

$5.2 \quad$ First-order Data-Passing Scheme . . . . . . . . . . . . . . . . 6

6 Stability ............................ 9

6.1 Stability of Defect approximation . . . . . . . . . . . . . . . 9

6.2 Stability of Correction Step of DDC . . . . . . . . . . . . . . . . 10

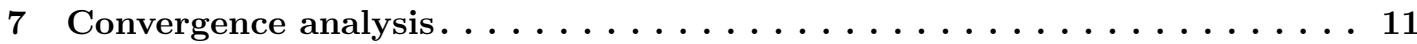

7.1 Accuracy of Defect Solution . . . . . . . . . . . . . . . . . . . . 11

7.2 Accuracy of Time Derivative of the Error in the Defect Step . . . . . . . . . . . 12

7.3 Accuracy of Correction Step . . . . . . . . . . . . . . . . . . . . . . . . 14

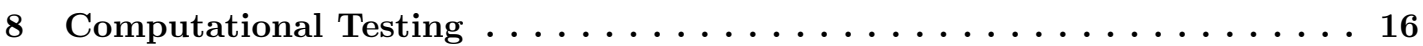

8.1 Convergence rate study $\ldots \ldots \ldots \ldots \ldots$

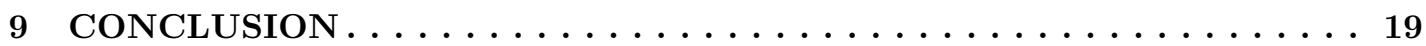

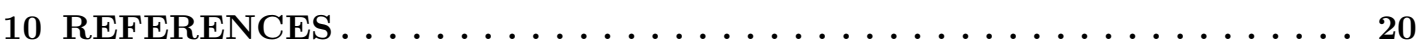




\section{List of Tables}

\section{List of Tables}

1 Errors for computed approximations, $\boldsymbol{\nu}=1$, non-modified jump condition $\ldots . \quad 17$

2 Errors for computed approximations, $\boldsymbol{\nu}=1$, modified jump condition . . . . . . 17

3 Errors for computed approximations, $\boldsymbol{\nu}=10^{-5}$, non-modified jump condition . 18

$4 \quad$ Errors for computed approximations, $\boldsymbol{\nu}=10^{-5}$, modified jump condition . . . . 18 


\section{Acknowledgements}

\section{ACKNOWLEDGEMENTS}

First, I would like to thank Assistant Professor Alexander Labovsky for his continuous support and advise.

I would also like to thank Associate Professor Aleksandr Sergeyev and Professor Allan Struthers for taking time out of their busy schedules in order to serve as my committee members.

Finally, the warmest gratitude goes to my family for their unconditional encouragement, patience and emotional support. 


\section{Abstract}

We present a method for solving a fluid-fluid interaction problem (two convection-dominated convection-diffusion problems adjoined by an interface), which is a simplified version of the atmosphere-ocean coupling problem. The method resolves some of the issues that can be crucial to the fluid-fluid interaction problems: it is a partitioned time stepping method, yet it is of high order accuracy in both space and time (the two-step algorithm considered in this report provides second order accuracy); it allows for the usage of the legacy codes (which is a common requirement when resolving flows in complex geometries), yet it can be applied to the problems with very small viscosity/diffusion coefficients. This is achieved by combining the defect correction technique for increased spatial accuracy (and for resolving the issue of high convection-to-diffusion ratio) with the deferred correction in time (which allows for the usage of the computationally attractive partitioned scheme, yet the time accuracy is increased beyond the usual result of partitioned methods being only first order accurate) into the defect-deferred correction method (DDC). The results are readily extendable to the higher order accuracy cases by adding more correction steps. Both the theoretical results and the numerical tests provided demonstrate that the computed solution is unconditionally stable and the accuracy in both space and time is improved after the correction step. 


\section{Introduction}

When attempting to solve a two-domain fluid-fluid interaction problem in a large domain with complex geometries, several key issues immediately appear. The more complicated the setting is, the more we are inclined to use the legacy codes - highly optimized black box subdomain solvers. This is often the only available option, because the monolithic, coupled problem can be difficult to efficiently discretize and solve. Thus, an attractive approach to some problems (as an ambitious underlying goal, consider the hurricane prediction, an atmosphereocean application on a huge domain with very complex boundaries and turbulent atmosphere flow) is the partitioned time stepping method which would decouple the problem and allow for the easy implementation of subdomain solvers. Additionally, these subdomain equations can be solved in parallel, if the data is explicitly passed across the shared interface at each time step.

Keeping with the goal of modelling the turbulent atmosphere-ocean flows using the preexisting codes for the atmosphere (separately) and the ocean, we seek, as a starting point of our project, an unconditionally stable partitioned time stepping method for fluid-fluid problems. Two of these methods, the IMEX method and the data-passing scheme, were proposed and thoroughly investigated in [15]; the data-passing scheme, introduced in this paper, was proven to be unconditionally stable for the two-domain heat-heat coupled problem. The same group of authors then successfully applied this method to the atmosphere-ocean coupled problem, proving that there exists a modification of the interface condition that allows for the unconditional stability of the data-passing scheme.

In this paper we aim at improving two existing flaws of this method: it is only first order accurate in space and time, and it is not designed for the turbulent (or convection-dominated) flows. Many turbulence models are available for the Navier-Stokes equations at high Reynolds numbers, but most of them do not allow for the usage of legacy codes (see, e.g., [20] for a deferred correction method combined with a turbulence model).

As a step towards the turbulent atmosphere-ocean coupling, we consider the two-domain convection-diffusion problem at high convection-to-diffusion ratio (in the computational tests we take the ratio of convection to diffusion coefficients to be $\left.10^{5}\right)$. The interface condition is the linearized version of the rigid-lid condition used in meteorology, see [14] for a more detailed discussion on the rigid-lid condition and the references therein. In order to create an unconditionally stable, second order accurate in both space and time, partitioned time stepping method, we apply the combined defect and deferred correction techniques to the data-passing scheme of [15]. The combination of the defect and deferred correction was introduced and 
successfully tested in [2] in application to the one-domain Navier-Stokes equations.

Consider the $d$-dimensional domain (in this report we consider $d=2$ ) $\Omega$ that consists of two subdomains $\Omega_{1}$ and $\Omega_{2}$ coupled across an interface $I$ (example in Figure 1 below).

The problem is: given $b_{i} \in \mathbb{R}^{d}, \nu_{i}>0, f_{i}:[0, T] \rightarrow H^{1}\left(\Omega_{i}\right)^{d}, u_{i}(0) \in H^{1}\left(\Omega_{i}\right)^{d}$ and $\kappa \in \mathbb{R}$, find (for $i=1,2) u_{i}: \Omega_{i} \times[0, T] \rightarrow \mathbb{R}^{d}$ satisfying

$$
\begin{aligned}
u_{i, t}-\nu_{i} \Delta u_{i}+b_{i} \cdot \nabla u_{i} & =f_{i}, \quad \text { in } \Omega_{i}, \\
-\nu_{i} \nabla u_{i} \cdot \hat{n}_{i} & =\kappa\left(u_{i}-u_{j}\right), \quad \text { on } I, i, j=1,2, i \neq j, \\
u_{i}(x, 0) & =u_{i}^{0}(x), \quad \text { in } \Omega_{i}, \\
u_{i} & =g_{i}, \quad \text { on } \Gamma_{i}=\partial \Omega_{i} \backslash I .
\end{aligned}
$$

Let

$$
X_{i}:=\left\{v_{i} \in H^{1}\left(\Omega_{i}\right)^{d}: v_{i}=0 \text { on } \Gamma_{i}\right\} .
$$

For $u_{i} \in X_{i}$ we denote $\mathbf{u}=\left(u_{1}, u_{2}\right), \mathbf{f}=\left(f_{1}, f_{2}\right)$ and $X:=\left\{\mathbf{v}=\left(v_{1}, v_{2}\right): v_{i} \in H^{1}\left(\Omega_{i}\right)^{d}\right.$ : $v_{i}=0$ on $\left.\Gamma_{i}, i=1,2\right\}$. A natural subdomain variational formulation for (4.1)-(4.4), obtained by multiplying (4.1) by $v_{i}$, integrating and applying the divergence theorem, is to find (for $i, j=1,2, i \neq j) u_{i}:[0, T] \rightarrow X_{i}$ satisfying

$$
\left(u_{i, t}, v_{i}\right)_{\Omega_{i}}+\nu_{i}\left(\nabla u_{i}, \nabla v_{i}\right)_{\Omega_{i}}+\int_{I} \kappa\left(u_{i}-u_{j}\right) v_{i} d s+\left(b_{i} \cdot \nabla u_{i}, v_{i}\right)_{\Omega_{i}}=\left(f_{i}, v_{i}\right)_{\Omega_{i}} \text {, for all } v_{i} \in X_{i} .
$$

The natural monolithic variational formulation for (4.1)-(4.4) is found by summing (4.5) over $i, j=1,2$ and $i \neq j$ and is to find $\mathbf{u}:[0, T] \rightarrow X$ satisfying

$$
\left(\mathbf{u}_{t}, \mathbf{v}\right)+\nu(\nabla \mathbf{u}, \nabla \mathbf{v})+\int_{I} \kappa[\mathbf{u}][\mathbf{v}] d s+(b \cdot \nabla \mathbf{u}, \mathbf{v})=(\mathbf{f}, \mathbf{v}), \forall v \in X,
$$

where [.] denotes the jump of the indicated quantity across the interface $I,(\cdot, \cdot)$ is the $L^{2}\left(\Omega_{1} \cup\right.$ $\left.\Omega_{2}\right)$ inner product and $\nu=\nu_{i}$ in $\Omega_{i}$.

Figure 1 illustrates the subdomains considered here, representative of commonly studied models in fluid-fluid and fluid-structure interaction, [10, 11, 15]. Comparing (4.6) and (4.5) we see that the monolithic problem (4.6) has a global energy that is exactly conserved, (in the appropriate sense), (set $\mathbf{v}=\mathbf{u}$ in (4.6)). The subdomain sub-problems (4.5) do not possess a subdomain energy which behaves similarly due to energy transfer back and forth across the interface $I$. It is possible for decoupling strategies to become unstable due to the input of non-physical energy as a numerical artifact.

Fluid-structure interaction problems, in particular blood flow models, are another typical application of partitioned methods. In these models the equations of elastic deformation of an arterial wall are coupled to equations of fluid flow through the vessel. Recently, it has 


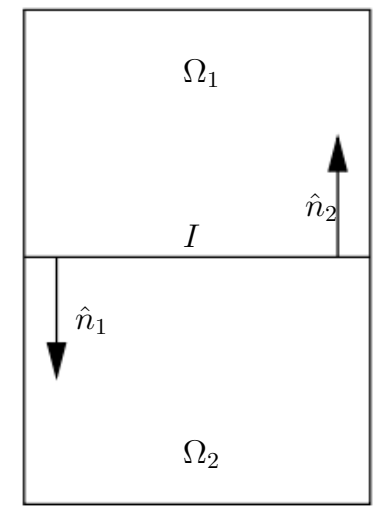

Figure 1: Example subdomains, coupled across an interface $I$.

been shown partitioned methods may be employed for this problem with the addition of a stabilization term on the fluid-structure interface. A defect correction step is implemented to recover optimal time accuracy, (see [11]).

In this report, a second order in space and time, non-overlapping uncoupling method for (4.1)-(4.4) is presented: the two-step Defect-Deferred Correction (DDC) method. At each step of the method the interface term in (4.5) is advanced in time to give one step black box decoupling of the subdomain problems in $\Omega_{1}$ and $\Omega_{2}$. Additionally, the deferred correction technique allows for the different time scales to be used in different domains, and even for the different terms within the same equation (see the work of Minion et al, [28, 7, 29] and the references for more details). This is important when the rapidly changing atmospheric flow is coupled the ocean flow that is changing at a much slower pace; also, the diffusion and the convection terms sometimes need to be modelled at different time scales.

The general idea of any Defect Correction Method (DCM) can be formulated as follows (see, e.g., $[30,8])$ :

Find a unique solution of $F x=0$, by

DCM: Use an approximation $\tilde{F}$ to build an iterative procedure:

$$
\begin{array}{r}
\tilde{F} x_{1}=0, \\
x_{i+1}=\left(I-\tilde{F}^{-1} F\right) x_{i}, i \geq 1 .
\end{array}
$$

The choice of a particular approximation $\tilde{F}$ determines the defect correction method in use. The general idea of defect correction and deferred correction methods for solving partial differential equations has been known for a long time, see the survey article [8]. Defect correction was proven computationally attractive in fluid applications. See, e.g., $[24,18,23,2,4,25]$ and references therein for other defect correction work relevant to fluids. 
The main advantage of the deferred correction approach is that a simple low-order method can be employed, and the recovered solution is of high-order accuracy, due to a sequence of deferred correction equations.

The classical deferred correction approach could be seen, e.g., in [19]. However, in 2000 a modification of the classical deferred correction approach was introduced by Dutt, Greengard and Rokhlin, [17]. This allowed the construction of stable and high-order accurate spectral deferred correction methods. In [27] M.L. Minion discusses these spectral deferred correction (SDC) methods in application to an initial value ODE

$$
\begin{aligned}
\phi^{\prime}(t) & =F(t, \phi(t)), t \in[a, b] \\
\phi(a) & =\phi_{a} .
\end{aligned}
$$

The solution is written in terms of the Picard integral equation; a polynomial is used to interpolate the subintegrand function and the obtained integral term is replaced by its quadrature approximation. In the case when the right hand side of the ODE can be decomposed into a sum of the stiff and non-stiff terms, a semi-implicit spectral deferred correction method (SISDC) is introduces, which allows to treat the non-stiff terms explicitly and the stiff terms implicitly. These SISDC methods for solving ordinary differential equations are further discussed in [27].

The remainder of this work is organized as follows: in Section 5, notation and mathematical preliminaries are given and the two-step defect-deferred correction method is introduced (Algorithm 5.1). The unconditional stability of the proposed method is proven in Section 6 . Convergence results are presented in Section 7, and computations are performed to investigate stability and accuracy of a two-step DDC algorithm in Section 8. 


\section{Method Description, Notation and Preliminaries}

This section presents the numerical schemes for (4.1)-(4.4), and provides the necessary definitions and lemmas for the stability and convergence analysis. For $D \subset \Omega$, the Sobolev space $H^{k}(D)=W^{k, 2}(D)$ is equipped with the usual norm $\|\cdot\|_{H^{k}(D)}$, and semi-norm $|\cdot|_{H^{k}(D)}$, for $1 \leq k<\infty$, e.g. Adams [1]. The $L^{2}$ norm is denoted by $\|\cdot\|_{D}$. For functions $v(x, t)$ defined for almost every $t \in(0, T)$ on a function space $V(D)$, we define the norms $(1 \leq p \leq \infty)$

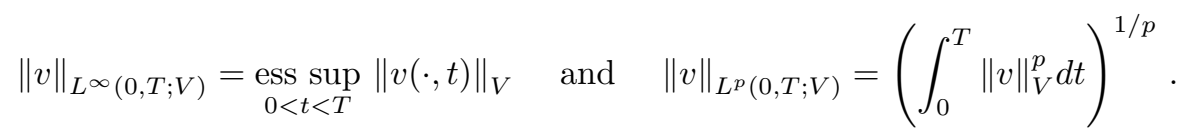

The dual space of the Banach space $V$ is denoted $V^{\prime}$.

Let the domain $\Omega \subset \mathbb{R}^{d}$ (typically $d=2,3$ ) have convex, polygonal subdomains $\Omega_{i}$ for $i=1,2$ with $\partial \Omega_{1} \cap \partial \Omega_{2}=\Omega_{1} \cap \Omega_{2}=I$. Let $\Gamma_{i}$ denote the portion of $\partial \Omega_{i}$ that is not on $I$, i.e. $\Gamma_{i}=\partial \Omega_{i} \backslash I$. For $i=1,2$, let $X_{i}=\left\{v \in H^{1}\left(\Omega_{i}\right)^{d}|v|_{\Gamma_{i}}=g_{i}\right\}$, let $(\cdot, \cdot)_{\Omega_{i}}$ denote the standard $L^{2}$ inner product on $\Omega_{i}$, and let $(\cdot, \cdot)_{X_{i}}$ denote the standard $H^{1}$ inner product on $\Omega_{i}$. Define $X=X_{1} \times X_{2}$ and $L^{2}(\Omega)=L^{2}\left(\Omega_{1}\right) \times L^{2}\left(\Omega_{2}\right)$. For $\mathbf{u}, \mathbf{v} \in X$ with $\mathbf{u}=\left[u_{1}, u_{2}\right]^{T}$ and $\mathbf{v}=\left[v_{1}, v_{2}\right]^{T}$, define the $L^{2}$ inner product

$$
(\mathbf{u}, \mathbf{v})=\sum_{i=1,2} \int_{\Omega_{i}} u_{i} v_{i} d x
$$

and $H^{1}$ inner product

$$
(\mathbf{u}, \mathbf{v})_{X}=\sum_{i=1,2}\left(\int_{\Omega_{i}} u_{i} v_{i} d x+\int_{\Omega_{i}} \nabla u_{i} \cdot \nabla v_{i} d x\right)
$$

and the induced norms $\|\mathbf{v}\|=(\mathbf{v}, \mathbf{v})^{1 / 2}$ and $\|\mathbf{v}\|_{X}=(\mathbf{v}, \mathbf{v})_{X}{ }^{1 / 2}$, respectively. The case where $g_{i}=0, i=1,2$ will be considered here, and can be easily extended to the case of nonhomogeneous Dirichlet conditions on $\partial \Omega_{i} \backslash I$.

Lemma 1. $\left(X,\|\cdot\|_{X}\right)$ is a Hilbert space.

Proof. The choice of boundary conditions for $X_{1}$ and $X_{2}$ will ensure $X_{i} \subset H^{1}\left(\Omega_{i}\right), i=1,2$ are closed subspaces. Hence by the definitions of $(\cdot, \cdot)_{X}$ and $\|\cdot\|_{X},\left(X,\|\cdot\|_{X}\right)$ is a Hilbert space.

The following discrete Gronwall's lemma and its modified version from [22] will be utilized in the subsequent analysis.

Lemma 2. (Gronwall's lemma) Let $k, M$, and $a_{\mu}, b_{\mu}, c_{\mu}, \gamma_{\mu}$, for integers $\mu>0$, be nonnegative numbers such that

$$
a_{n}+k \sum_{\mu=0}^{n} b_{\mu} \leq k \sum_{\mu=0}^{n} \gamma_{\mu} a_{\mu}+k \sum_{\mu=0}^{n} c_{\mu}+M \text { for } n \geq 0 .
$$

Suppose that $k \gamma_{\mu}<1$, for all $\mu$, and set $\sigma_{\mu} \equiv\left(1-k \gamma_{\mu}\right)^{-1}$. Then,

$$
a_{n}+k \sum_{\mu=0}^{n} b_{\mu} \leq \exp \left(k \sum_{\mu=0}^{n} \sigma_{\mu} \gamma_{\mu}\right)\left\{k \sum_{\mu=0}^{n} c_{\mu}+M\right\} \text { for } n \geq 0 .
$$


The restriction on the time step can be waived if $\gamma_{n}=0$.

Lemma 3. (Modified Gronwall's lemma) Let $k, M$, and $a_{\mu}, b_{\mu}, c_{\mu}, \gamma_{\mu}$, for integers $\mu>0$, be nonnegative numbers such that

$$
a_{n}+k \sum_{\mu=0}^{n} b_{\mu} \leq k \sum_{\mu=0}^{n-1} \gamma_{\mu} a_{\mu}+k \sum_{\mu=0}^{n} c_{\mu}+M \text { for } n \geq 0
$$

Then, with $\sigma_{\mu} \equiv\left(1-k \gamma_{\mu}\right)^{-1}$,

$$
a_{n}+k \sum_{\mu=0}^{n} b_{\mu} \leq \exp \left(k \sum_{\mu=0}^{n-1} \sigma_{\mu} \gamma_{\mu}\right)\left\{k \sum_{\mu=0}^{n} c_{\mu}+M\right\} \text { for } n \geq 0
$$

\subsection{Discrete Formulation}

Let $\mathcal{T}_{i}$ be a triangulation of $\Omega_{i}$ and $\mathcal{T}_{h}=\mathcal{T}_{1} \cup \mathcal{T}_{2}$. Take $X_{i}^{h} \subset X_{i}$ to be conforming finite element spaces for $i=1,2$, and define $X^{h}=X_{1}^{h} \times X_{2}^{h} \subset X$. It follows that $X^{h} \subset X$ is a Hilbert

space with corresponding inner product and induced norm. We shall consider $X_{i}^{h}$ to be spaces of continuous piecewise polynomials of degree $m \geq 2$.

For $t_{k} \in[0, T], \mathbf{u}^{k}$ will denote the discrete approximation to $\mathbf{u}\left(t_{k}\right)$.

A partitioned time stepping approach for the heat-heat equations in the same setting as (4.1)-(4.4) was introduced by Connors, Howell, Layton in [15]. The analogue of this datapassing scheme for our problem is presented below.

\subsection{First-order Data-Passing Scheme}

Let $\Delta t>0, f_{i} \in L^{2}\left(\Omega_{i}\right)$. For each $M \in \mathbb{N}, M \leq \frac{T}{\Delta t}$, given $u_{i}^{n} \in X_{i, h}, n=0,1,2, \cdots, M-1$, solve on each subdomain (for $i, j=1,2, i \neq j$ ) to find $u_{i}^{n+1} \in X_{i, h}$ satisfying

$$
\begin{array}{rl}
\left(\frac{u_{i}^{n+1}-u_{i}^{n}}{\Delta t}, v_{i}\right)+\nu_{i}\left(\nabla u_{i}^{n+1}, \nabla v_{i}\right)+\kappa \int_{I}\left(u_{i}^{n+1}-u_{j}^{n}\right) v_{i} & d s+\left(b_{i} \cdot \nabla u_{i}^{n}, v_{i}\right) \\
=\left(f_{i}\left(t^{n+1}\right), v_{i}\right), \forall v_{i} \in X_{i, h}
\end{array}
$$

This scheme was extensively studied in [15] and was proven to be unconditionally stable and first order accurate. Moreover, in [14] the authors were able to extend this scheme to the atmosphere-ocean problem and prove (using a subtle modification of the jump condition) that the unconditional stability stands.

Based on this scheme, we now introduce the defect-deferred algorithm to increase the method's accuracy and expand the set of applications to include the flows at very high convectionto-diffusion ratio. The artificial viscosity is chosen to be the first order accurate spatial approximation to stabilize the convection-dominated flows; the defect correction algorithm (4.7) is then combined with the spectral deferred correction approach of [28]. 
Throughout the remainder of this paper we will use $t u, u, c u$ to denote, respectively, the true solution, the defect step approximation and the correction step approximation.

The defect correction method, based on the artificial viscosity approximation of (5.5), would lead to the following system of equations.

$$
\begin{gathered}
\left(\frac{u_{i}^{n+1}-u_{i}^{n}}{\Delta t}, v_{i}\right)+\left(\nu_{i}+h\right)\left(\nabla u_{i}^{n+1}, \nabla v_{i}\right)+\left(1+\frac{h}{\nu_{i}}\right) \kappa \int_{I}\left(u_{i}^{n+1}-u_{j}^{n}\right) v_{i} d s+\left(b_{i} \cdot \nabla u_{i}^{n}, v_{i}\right) \\
=\left(f_{i}\left(t^{n+1}\right), v_{i}\right), \forall v_{i} \in X_{i, h} \\
\left(\frac{c u_{i}^{n+1}-c u_{i}^{n}}{\Delta t}, v_{i}\right)+\left(\nu_{i}+h\right)\left(\nabla c u_{i}^{n+1}, \nabla v_{i}\right)+\left(1+\frac{h}{\nu_{i}}\right) \kappa \int_{I}\left(c u_{i}^{n+1}-c u_{j}^{n}\right) v_{i} d s+\left(b_{i} \cdot \nabla c u_{i}^{n}, v_{i}\right) \\
=\left(f_{i}\left(t^{n+1}\right), v_{i}\right)+h\left(\nabla u_{i}^{n+1}, \nabla v_{i}\right)+\frac{h}{\nu_{i}} \kappa \int_{I}\left(u_{i}^{n+1}-u_{j}^{n}\right) v_{i} d s, \forall v_{i} \in X_{i, h} .
\end{gathered}
$$

However, if the interface condition (4.2) is modified to replace $\nu_{i}$ with $\nu_{i}+h$, this results in

$$
\begin{array}{r}
\left(\frac{u_{i}^{n+1}-u_{i}^{n}}{\Delta t}, v_{i}\right)+\left(\nu_{i}+h\right)\left(\nabla u_{i}^{n+1}, \nabla v_{i}\right)+\kappa \int_{I}\left(u_{i}^{n+1}-u_{j}^{n}\right) v_{i} d s+\left(b_{i} \cdot \nabla u_{i}^{n}, v_{i}\right) \\
=\left(f_{i}\left(t^{n+1}\right), v_{i}\right), \forall v_{i} \in X_{i, h} \\
\left(\frac{c u_{i}^{n+1}-c u_{i}^{n}}{\Delta t}, v_{i}\right)+\left(\nu_{i}+h\right)\left(\nabla c u_{i}^{n+1}, \nabla v_{i}\right)+\kappa \int_{I}\left(c u_{i}^{n+1}-c u_{j}^{n}\right) v_{i} d s+\left(b_{i} \cdot \nabla c u_{i}^{n}, v_{i}\right) \\
=\left(f_{i}\left(t^{n+1}\right), v_{i}\right)+h\left(\nabla u_{i}^{n+1}, \nabla v_{i}\right), \forall v_{i} \in X_{i, h} .
\end{array}
$$

Both the modified and non-modified jump conditions were compared numerically, in favor of (5.6) (see Section 8). Therefore, only the theory for this approach will be considered below.

The deferred correction algorithm applied to the model problem (5.5) is as follows.

$$
\begin{array}{r}
\left(\frac{u_{i}^{n+1}-u_{i}^{n}}{\Delta t}, v_{i}\right)+\nu_{i}\left(\nabla u_{i}^{n+1}, \nabla v_{i}\right)+\kappa \int_{I}\left(u_{i}^{n+1}-u_{j}^{n}\right) v_{i} d s+\left(b_{i} \cdot \nabla u_{i}^{n}, v_{i}\right) \\
=\left(f_{i}\left(t^{n+1}\right), v_{i}\right), \forall v_{i} \in X_{i}^{h} \\
\left(\frac{c u_{i}^{n+1}-c u_{i}^{n}}{\Delta t}, v_{i}\right)+\nu_{i}\left(\nabla r_{i}^{n+1}, \nabla v_{i}\right)+\kappa \int_{I}\left(r_{i}^{n+1}-r_{j}^{n}\right) v_{i} d s+\left(b_{i} \cdot \nabla r_{i}^{n}, v_{i}\right)=\frac{1}{\Delta t} I_{n}^{n+1}\left(u_{i}\right) .
\end{array}
$$

Here $r_{i}^{k}=c u_{i}^{k}-u_{i}^{k}, k=0,1, \ldots, N$.

$I_{n}^{n+1}\left(u_{i}\right)$ is a numerical quadrature approximation to $\int_{t_{n}}^{t_{n+1}} F\left(\tau, u_{i}(\tau)\right) d \tau$, where $F\left(t, u_{i}\right)=$ $\left(f_{i}(t), v_{i}\right)-\nu_{i}\left(\nabla u_{i}(t), v_{i}\right)+\kappa \int_{I}\left(u_{i}(t)-u_{j}(t)\right) v_{i} d s+\left(b_{i} \cdot \nabla u_{i}(t), v_{i}\right)$.

Remark 5.1. Provided the integral terms $I_{n}^{n+1}\left(u_{i}\right)$ are computed with the accuracy of order $O\left((\Delta t)^{2}\right)$, after 1 correction iteration the above procedure will produce an approximate solution with global accuracy $O\left((\Delta t)^{2}\right)$. If the points $t_{m} \in\left[t_{n}, t_{n+1}\right]$ are chosen to be Gaussian quadrature nodes, then the integral is being computed with a spectral integration rule, which is the reason for the name spectral deferred corrections. For the two-step method the spectral integration simplifies to the trapezoid rule. 
The variational formulation of the two-step Defect Deferred Correction methods is obtained by combining the defect and deferred correction techniques (5.6)-(5.7) into the following

Algorithm 5.1 (Two Step DDC). Let $\Delta t>0, M=\frac{T}{\Delta t}, f_{i} \in L^{2}\left(\Omega_{i}\right)$. Given $u_{i}^{n}$, find $u_{i}^{n+1} \in X_{i}^{h}, i, j=1,2, i \neq j, n=0,1,2, \cdots, M-1$, satisfying

$$
\begin{array}{r}
\left(\frac{u_{i}^{n+1}-u_{i}^{n}}{\Delta t}, v_{i}\right)+\left(\nu_{i}+h\right)\left(\nabla u_{i}^{n+1}, \nabla v_{i}\right)+\kappa \int_{I}\left(u_{i}^{n+1}-u_{j}^{n}\right) v_{i} d s+\left(b_{i} \cdot \nabla u_{i}^{n}, v_{i}\right) \\
=\left(f_{i}^{n+1}, v_{i}\right), \forall v_{i} \in X_{i, h}
\end{array}
$$

Also, given $c u_{i}^{n}$, find $c u_{i}^{n+1} \in X_{i}^{h}$ satisfying

$$
\begin{gathered}
\left(\frac{c u_{i}^{n+1}-c u_{i}^{n}}{\Delta t}, v_{i}\right)+\left(\nu_{i}+h\right)\left(\nabla c u_{i}^{n+1}, \nabla v_{i}\right)+\kappa \int_{I}\left(c u_{i}^{n+1}-c u_{j}^{n}\right) v_{i} d s+\left(b \cdot \nabla c u_{i}^{n}, v_{i}\right) \\
=\left(\frac{f_{i}^{n+1}+f_{i}^{n}}{2}, v_{i}\right)-\frac{\Delta t}{2}\left(b_{i} \cdot \nabla\left(\frac{u_{i}^{n+1}-u_{i}^{n}}{\Delta t}\right), v_{i}\right)+\Delta t \frac{\left(\nu_{i}+h\right)}{2}\left(\nabla\left(\frac{u_{i}^{n+1}-u_{i}^{n}}{\Delta t}\right), \nabla v_{i}\right) \\
+\frac{\kappa}{2} \Delta t \int_{I}\left(\frac{u_{i}^{n+1}-u_{i}^{n}}{\Delta t}\right) v_{i} d s+\frac{\kappa}{2} \Delta t \int_{I}\left(\frac{u_{j}^{n+1}-u_{j}^{n}}{\Delta t}\right) v_{i} d s+h\left(\nabla\left(\frac{u_{i}^{n+1}+u_{i}^{n}}{2}\right), \nabla v_{i}\right), \forall v_{i} \in X_{i, h} .
\end{gathered}
$$

The terms in the right hand side of (5.9) are written in a form that hints at the reason for the increased accuracy of the correction step solution. Note also that the structure of the left hand side (and therefore the matrix of the system) is identical for (5.8) and (5.9); thus, a simple and computationally cheap artificial viscosity data-passing approximation is computed twice to achieve higher accuracy while maintaining the unconditional stability. 


\section{Stability}

In this section we prove the unconditional stability of both the defect step and the correction step approximations.

\subsection{Stability of Defect approximation}

Lemma 4. (Stability of Defect approximation) Let $\mathbf{u}^{n+1} \in X^{h}$ satisfy (5.8) for each $n \in$ $\left\{0,1,2, \cdots, \frac{T}{\Delta t}-1\right\}$. Then $\exists C>0$ independent of $h, \Delta t$ such that $\mathbf{u}^{n+1}$ satisfies:

$$
\begin{aligned}
& \left\|\mathbf{u}^{n+1}\right\|^{2}+(\nu+h) \Delta t \sum_{k=1}^{n+1}\left\|\nabla \mathbf{u}^{k}\right\|^{2}+\kappa \Delta t\left(\left\|u_{1}^{n+1}\right\|_{I}^{2}+\left\|u_{2}^{n+1}\right\|_{I}^{2}\right) \\
& \leq C\left\{\left\|\mathbf{u}^{0}\right\|^{2}+\kappa \Delta t\left(\left\|u_{1}^{0}\right\|_{I}^{2}+\left\|u_{2}^{0}\right\|_{I}^{2}\right)+\frac{2}{\nu+h} \Delta t \sum_{k=1}^{n+1}\left\|\mathbf{f}^{k}\right\|_{-1}^{2}\right\} .
\end{aligned}
$$

Proof. Choose $v_{i}=u_{i}^{n+1}$ in (5.8), $i \neq j$ to obtain

$$
\begin{aligned}
\left(\frac{u_{i}^{n+1}-u_{i}^{n}}{\Delta t}, u_{i}^{n+1}\right)+(\nu+h) \| \nabla & u_{i}^{n+1} \|^{2}+\left(b \cdot \nabla u_{i}^{n}, u_{i}^{n+1}\right) \\
& +\int_{I} \kappa\left(u_{i}^{n+1}-u_{j}^{n}\right) u_{i}^{n+1} d s=\left(f_{i}^{n+1}, u_{i}^{n+1}\right) .
\end{aligned}
$$

Applying the Cauchy-Schwarz inequality and summing over $\mathrm{i}, \mathrm{j}=1,2, i \neq j$, yields

$$
\begin{array}{r}
\frac{\left\|\mathbf{u}^{n+1}\right\|^{2}-\left\|\mathbf{u}^{n}\right\|^{2}}{2 \Delta t}+(\nu+h)\left\|\nabla \mathbf{u}^{n+1}\right\|^{2}+\left(b \cdot \nabla \mathbf{u}^{n}, \mathbf{u}^{n+1}\right)+\kappa\left\|u_{1}^{n+1}\right\|_{I}^{2}+\kappa\left\|u_{2}^{n+1}\right\|_{I}^{2} \\
-\kappa\left\|u_{1}^{n}\right\|_{I}\left\|u_{2}^{n+1}\right\|_{I}-\kappa\left\|u_{2}^{n}\right\|_{I}\left\|u_{1}^{n+1}\right\|_{I} \leq\left\|\nabla \mathbf{u}^{n+1}\right\|\left\|\mathbf{f}^{n+1}\right\|_{-1} .
\end{array}
$$

Young's inequality allows to "hide" all the u-terms, leading to the telescoping series in the left hand side (LHS)

$$
\begin{array}{r}
\frac{\left\|\mathbf{u}^{n+1}\right\|^{2}-\left\|\mathbf{u}^{n}\right\|^{2}}{2 \Delta t}+(\nu+h)\left\|\nabla \mathbf{u}^{n+1}\right\|^{2}+\frac{\kappa}{2}\left(\left\|u_{1}^{n+1}\right\|_{I}^{2}-\left\|u_{1}^{n}\right\|_{I}^{2}\right)+\frac{\kappa}{2}\left(\left\|u_{2}^{n+1}\right\|_{I}^{2}-\left\|u_{2}^{n}\right\|_{I}^{2}\right) \\
\leq \frac{\nu+h}{4}\left\|\nabla \mathbf{u}^{n+1}\right\|^{2}+\frac{1}{\nu+h}\left\|\mathbf{f}^{n+1}\right\|_{-1}^{2}+\frac{\nu+h}{4}\left\|\nabla \mathbf{u}^{n+1}\right\|^{2}+\frac{|b|^{2}}{\nu+h}\left\|\mathbf{u}^{n}\right\|^{2}
\end{array}
$$

Summing over the time levels and multiplying by $2 \Delta t$, we obtain

$$
\begin{array}{r}
\left\|\mathbf{u}^{n+1}\right\|^{2}+(\nu+h) \Delta t \sum_{k=1}^{n+1}\left\|\nabla \mathbf{u}^{k}\right\|^{2}+\kappa \Delta t\left(\left\|u_{1}^{n+1}\right\|_{I}^{2}+\left\|u_{2}^{n+1}\right\|_{I}^{2}\right) \\
\leq\left\|\mathbf{u}^{0}\right\|^{2}+\kappa \Delta t\left(\left\|u_{1}^{0}\right\|_{I}^{2}+\left\|u_{2}^{0}\right\|_{I}^{2}\right)+\frac{2}{\nu+h} \Delta t \sum_{k=1}^{n+1}\left\|\mathbf{f}^{k}\right\|_{-1}^{2}+\frac{2|b|^{2}}{\nu+h} \Delta t \sum_{k=0}^{n}\left\|\mathbf{u}^{k}\right\|^{2}
\end{array}
$$

The summation in the last term on the right hand side does not include the time level $(n+1)$. Therefore, using the modified Gronwall's Lemma, we obtain

$$
\begin{gathered}
\left\|\mathbf{u}^{n+1}\right\|^{2}+(\nu+h) \Delta t \sum_{k=1}^{n+1}\left\|\nabla \mathbf{u}^{k}\right\|^{2}+\kappa \Delta t\left(\left\|u_{1}^{n+1}\right\|_{I}^{2}+\left\|u_{2}^{n+1}\right\|_{I}^{2}\right) \\
\leq C\left\{\left\|\mathbf{u}^{0}\right\|^{2}+\kappa \Delta t\left(\left\|u_{1}^{0}\right\|_{I}^{2}+\left\|u_{2}^{0}\right\|_{I}^{2}\right)+\frac{2}{\nu+h} \Delta t \sum_{k=1}^{n+1}\left\|\mathbf{f}^{k}\right\|_{-1}^{2}\right\}
\end{gathered}
$$

Hence, the initial approximation $\mathbf{u}$ is unconditionally stable. We conclude the proof of stability of the DDC approximations by considering the second step approximation $\mathbf{c u}$. 
6.2. Stability of Correction Step of DDC

Theorem 5 (Stability of Correction Step of DDC). Let $\mathbf{c u}^{\mathbf{n}+\mathbf{1}} \in \mathbf{X}^{\mathbf{h}}$ satisfy (5.9) for each $n \in\left\{0,1,2, \cdots, \frac{T}{\Delta t}-1\right\}$. Then $\exists C>0$ independent of $h, \Delta t$ such that $\mathbf{c u}^{n+1}$ satisfies:

$$
\begin{array}{r}
\left\|\mathbf{c} \mathbf{u}^{n+1}\right\|^{2}+(\nu+h) \Delta t \sum_{k=1}^{n+1}\left\|\nabla \mathbf{c} \mathbf{u}^{k}\right\|^{2}+\kappa \Delta t\left(\left\|c u_{1}^{n+1}\right\|_{I}^{2}+\left\|c u_{2}^{n+1}\right\|_{I}^{2}\right) \leq C\left[\left\|\mathbf{c u}^{0}\right\|^{2}+\kappa \Delta t\left(\left\|c u_{1}^{0}\right\|_{I}^{2}\right.\right. \\
\left.\left.+\left\|c u_{2}^{0}\right\|_{I}^{2}\right)+\frac{1}{\nu+h}\left\{\left\|\mathbf{u}^{0}\right\|^{2}+\kappa \Delta t\left(\left\|u_{1}^{0}\right\|_{I}^{2}+\left\|u_{2}^{0}\right\|_{I}^{2}\right)+\frac{1}{\nu+h} \Delta t \sum_{k=1}^{n+1}\left\|\mathbf{f}^{k}\right\|_{-1}^{2}\right\}\right] .
\end{array}
$$

Proof. Choosing $v_{i}=c u_{i}^{n+1}$ in (5.9) gives

$$
\begin{aligned}
& \left(\frac{c u_{i}^{n+1}-c u_{i}^{n}}{\Delta t}, c u_{i}^{n+1}\right)+\left(\nu_{i}+h\right)\left\|\nabla c u_{i}^{n+1}\right\|^{2}+\left(b_{i} \cdot \nabla c u_{i}^{n}, c u_{i}^{n+1}\right) \\
& +\int_{I} \kappa\left(c u_{i}^{n+1}-c u_{j}^{n}\right) c u^{n+1} d s=\left(\frac{f_{i}^{n+1}+f_{i}^{n}}{2}, c u_{i}^{n+1}\right)-\frac{\Delta t}{2}\left(b \cdot \nabla\left(\frac{u_{i}^{n+1}-u_{i}^{n}}{\Delta t}\right), c u_{i}^{n+1}\right) \\
& +\frac{\Delta t\left(\nu_{i}+h\right)}{2}\left(\nabla\left(\frac{u_{i}^{n+1}-u_{i}^{n}}{\Delta t}\right), \nabla c u_{i}^{n+1}\right)+\frac{1}{2} \kappa \int_{I}\left(u_{j}^{n+1}-u_{j}^{n}+u_{i}^{n+1}-u_{i}^{n}\right) c u_{i}^{n+1} d s \\
& +h\left(\nabla\left(\frac{u_{i}^{n+1}+u_{i}^{n}}{2}\right), \nabla c u_{i}^{n+1}\right) .
\end{aligned}
$$

Compared to the proof of stability of the defect solution $\mathbf{u}$, there are four extra terms in the RHS. They are bounded as follows. After the summation over $i=1,2$ we obtain

$$
\begin{aligned}
\mid \frac{\Delta t}{2}\left(b \cdot \nabla\left(\frac{\mathbf{u}^{n+1}-\mathbf{u}^{n}}{\Delta t}\right),\right. & \left.\mathbf{c u} \mathbf{u}^{n+1}\right)\left|\leq \frac{1}{2}\right|\left(b \cdot \nabla \mathbf{c} \mathbf{u}^{n+1}, \mathbf{u}^{n+1}\right)\left|+\frac{1}{2}\right|\left(b \cdot \nabla \mathbf{c u} \mathbf{u}^{n+1}, \mathbf{u}^{n}\right) \mid \\
& \leq 2 \epsilon(\nu+h)\left\|\nabla \mathbf{c u}^{n+1}\right\|^{2}+\frac{|b|^{2}}{16 \epsilon(\nu+h)}\left(\left\|\mathbf{u}^{n+1}\right\|^{2}+\left\|\mathbf{u}^{n}\right\|^{2}\right) .
\end{aligned}
$$

Similarly

$$
\begin{aligned}
& \left|\frac{\nu+h}{2}\left(\nabla \mathbf{u}^{n+1}, \nabla \mathbf{c u}{ }^{n+1}\right)-\frac{\nu+h}{2}\left(\nabla u^{n}, \nabla \mathbf{c u}^{n+1}\right)\right| \leq 2 \epsilon(\nu+h)\left\|\nabla \mathbf{c u}^{n+1}\right\|^{2} \\
& +\frac{\nu+h}{16 \epsilon}\left(\left\|\nabla \mathbf{u}^{n+1}\right\|^{2}+\left\|\nabla \mathbf{u}^{n}\right\|^{2}\right) .
\end{aligned}
$$

Using again the Cauchy-Swcharz and Young's inequalities, we find a bound on the $h$-term as follows

$$
\left|h\left(\nabla\left(\frac{\mathbf{u}^{n+1}+\mathbf{u}^{n}}{2}\right), \nabla \mathbf{c} \mathbf{u}^{n+1}\right)\right| \leq 2 \epsilon(\nu+h)\left\|\nabla \mathbf{c u}^{n+1}\right\|^{2}+\frac{h^{2}}{16 \epsilon(\nu+h)}\left(\left\|\nabla \mathbf{u}^{n+1}\right\|^{2}+\left\|\nabla \mathbf{u}^{n}\right\|^{2}\right) .
$$

Summing over the time levels, choosing $\epsilon=\frac{1}{28}$ allows us to hide the $\nabla \mathbf{c u}$-terms in the LHS. There are also eight boundary terms

$$
\frac{\kappa}{2} \int_{I}\left(u_{2}^{n+1}-u_{2}^{n}+u_{1}^{n+1}-u_{1}^{n}\right) c u_{1}^{n+1} d s+\frac{\kappa}{2} \int_{I}\left(u_{1}^{n+1}-u_{1}^{n}+u_{2}^{n+1}-u_{2}^{n}\right) c u_{2}^{n+1} d s .
$$

For each of the eight terms, use the Cauchy-Schwarz inequality, followed by the Young's inequality and use the trace theorem to obtain the bounds

$$
\begin{aligned}
\left\|c u_{i}^{n+1}\right\|_{I}^{2} \leq\left\|c u_{i}^{n+1}\right\|_{\partial \Omega_{i}}^{2} & \leq C_{\text {Trace }}\left\|\nabla c u_{i}^{n+1}\right\|_{\Omega_{i}}^{2} \\
\left\|u_{i}^{n+1}\right\|_{I}^{2} \leq\left\|u_{i}^{n+1}\right\|_{\partial \Omega_{i}}^{2} & \leq C_{\text {Trace }}\left\|\nabla u_{i}^{n+1}\right\|_{\Omega_{i}}^{2} .
\end{aligned}
$$

Utilizing the stability bound on the defect solution $\mathbf{u}$ completes the proof. 


\section{Convergence analysis}

We start by proving the accuracy estimate of the defect solution.

\subsection{Accuracy of Defect Solution}

Theorem 6. (Accuracy of Defect Solution) Let $t u_{i}(t ; x) \in L^{2}(0, T ; X)$ solve (4.1)-(4.4) for all $t \in(0, T)$. Let also $t u_{i, t}(t ; x) \in L^{2}(0, T ; X)$ and $t u_{i, t t}(t ; x) \in L^{2}\left(0, T ; L^{2}\left(\Omega_{i}\right)\right), i=1,2$. Then $\exists C>0$ independent of $h, \Delta t$ such that for any $n \in\left\{0,1,2, \cdots, M-1=\frac{T}{\Delta t}-1\right\}$, the solution $u_{i}^{n+1}$ of (5.8) satisfies

$$
\left\|\mathbf{t} \mathbf{u}^{n+1}-\mathbf{u}^{n+1}\right\|^{2}+(\nu+h) \Delta t \sum_{j=1}^{n+1}\left\|\nabla\left(\mathbf{t} \mathbf{u}^{j}-\mathbf{u}^{j}\right)\right\|^{2}+\kappa \Delta t \sum_{i=1}^{2}\left\|t u_{i}^{n+1}-u_{i}^{n+1}\right\|_{I}^{2} \leq C\left(h^{2}+\Delta t^{2}\right)
$$

Proof. Restricting the test functions to $X_{h}$, write (4.5) at time $t_{n+1}$ as

$$
\begin{gathered}
\left(\frac{t u_{i}^{n+1}-t u_{i}^{n}}{\Delta t}, v_{i}\right)_{\Omega_{i}}+\left(\nu_{i}+h\right)\left(\nabla t u_{i}^{n+1}, \nabla v_{i}\right)_{\Omega_{i}}+\kappa \int_{I}\left(t u_{i}^{n+1}-t u_{j}^{n}\right) v_{i} d s+\left(b_{i} \cdot \nabla t u_{i}^{n}, v_{i}\right)_{\Omega_{i}} \\
=\left(f_{i}^{n+1}, v_{i}\right)_{\Omega_{i}}+h\left(\nabla t u_{i}^{n+1}, \nabla v_{i}\right)_{\Omega_{i}}+\left(\frac{t u_{i}^{n+1}-t u_{i}^{n}}{\Delta t}-t u_{i, t}^{n+1}, v_{i}\right)_{\Omega_{i}} \\
-\Delta t\left(b_{i} \cdot \nabla\left(\frac{t u_{i}^{n+1}-t u_{i}^{n}}{\Delta t}\right), v_{i}\right)_{\Omega_{i}}+\kappa \int_{I}\left(t u_{j}^{n+1}-t u_{j}^{n}\right) v_{i} d s
\end{gathered}
$$

Denote $\frac{t u_{i}^{n+1}-t u_{i}^{n}}{\Delta t}-t u_{i, t}^{n+1} \equiv \rho_{i}^{n+1}$. Subtract (5.8) from (7.2) to obtain the equation for the error, $e_{i}^{n+1}=t u_{i}^{n+1}-u_{i}^{n+1}, i=1,2$. For any $v_{i} \in X_{i}^{h}$

$$
\begin{array}{r}
\left(\frac{e_{i}^{n+1}-e_{i}^{n}}{\Delta t}, v_{i}\right)_{\Omega_{i}}+\left(\nu_{i}+h\right)\left(\nabla e_{i}^{n+1}, \nabla v_{i}\right)_{\Omega_{i}}+\kappa \int_{I}\left(e_{i}^{n+1}-e_{j}^{n}\right) v_{i} d s+\left(b_{i} \cdot \nabla e_{i}^{n}, v_{i}\right)_{\Omega_{i}} \\
=h\left(\nabla t u_{i}^{n+1}, \nabla v_{i}\right)_{\Omega_{i}}+\left(\rho_{i}^{n+1}, v_{i}\right)_{\Omega_{i}}-\Delta t\left(b_{i} \cdot \nabla\left(\frac{t u_{i}^{n+1}-t u_{i}^{n}}{\Delta t}\right), v_{i}\right) \\
+\Delta t \kappa \int_{I}\left(\frac{t u_{j}^{n+1}-t u_{j}^{n}}{\Delta t}\right) v_{i} d s, i \neq j .
\end{array}
$$

Do the summation over $i=1,2$; decompose the error $\mathbf{e}^{n+1}=\left(\tilde{\mathbf{u}}^{n+1}-\mathbf{u}^{n+1}\right)-\left(\tilde{\mathbf{u}}^{n+1}-\mathbf{t u}^{n+1}\right)=$ $\phi^{n+1}-\boldsymbol{\eta}^{n+1}$, for some $\tilde{\mathbf{u}}^{n+1} \in X_{h}$ and take $\mathbf{v}=\phi^{n+1} \in X^{h}$. Then $\forall n \geq 0$

$$
\begin{array}{r}
\left(\frac{\phi^{n+1}-\phi^{n}}{\Delta t}, \phi^{n+1}\right)+(\nu+h)\left(\nabla \phi^{n+1}, \nabla \phi^{n+1}\right)+\kappa \sum_{i=1,2} \int_{I}\left(\phi_{i}^{n+1}\right)^{2} d s=h\left(\nabla t u^{n+1}, \nabla \phi^{n+1}\right) \\
+\left(\rho^{n+1}, \phi^{n+1}\right)+\Delta t \kappa \int_{I}\left(\frac{t u^{n+1}-t u^{n}}{\Delta t}\right) \phi^{n+1} d s+\left(\frac{\boldsymbol{\eta}^{n+1}-\boldsymbol{\eta}^{n}}{\Delta t}, \boldsymbol{\phi}^{n+1}\right)+(\nu+h)\left(\nabla \boldsymbol{\eta}^{n+1}, \nabla \boldsymbol{\phi}^{n+1}\right) \\
-\Delta t\left(b_{i} \cdot \nabla\left(\frac{t u_{i}^{n+1}-t u_{i}^{n}}{\Delta t}\right), \phi^{n+1}\right)+\kappa \int_{I} \phi^{n+1} \boldsymbol{\eta}^{n+1} d s+\kappa \sum_{i \neq j} \int_{I} \mathbf{e}_{j}^{n} \boldsymbol{\phi}_{i}^{n+1} d s+\left(b \cdot \nabla \boldsymbol{\eta}^{n}, \boldsymbol{\phi}^{n+1}\right) \\
-\left(b \cdot \nabla \boldsymbol{\phi}^{n}, \boldsymbol{\phi}^{n+1}\right)+\frac{|b|^{2}}{4 \epsilon(\nu+h)}(\Delta t)^{2}\left\|\nabla\left(\frac{\mathbf{t u}^{n+1}-\mathbf{t u}^{n}}{\Delta t}\right)\right\|^{2}
\end{array}
$$


Using the Cauchy-Schwarz and Young's inequalities followed by the Trace theorem gives

$$
\begin{gathered}
\frac{\left\|\boldsymbol{\phi}^{n+1}\right\|^{2}-\left\|\boldsymbol{\phi}^{n}\right\|^{2}}{2 \Delta t}+(\nu+h)\left(\left\|\nabla \boldsymbol{\phi}^{n+1}\right\|^{2}+\kappa\left\|\boldsymbol{\phi}_{1}^{n+1}\right\|_{I}^{2}+\kappa\left\|\boldsymbol{\phi}_{2}^{n+1}\right\|_{I}^{2} \leq \epsilon(\nu+h)\left\|\nabla \boldsymbol{\phi}^{n+1}\right\|^{2}\right. \\
+\frac{h^{2}}{4 \epsilon(\nu+h)}\left\|\nabla \mathbf{t} \mathbf{u}^{n+1}\right\|^{2}+\epsilon(\nu+h)\left\|\nabla \boldsymbol{\phi}^{n+1}\right\|^{2}+\frac{C_{P F}^{2}}{4 \epsilon(\nu+h)}\left\|\rho^{n+1}\right\|^{2}+\epsilon(\nu+h)\left\|\nabla \boldsymbol{\phi}^{n+1}\right\|^{2} \\
+\frac{C_{T r a c e}^{4} \Delta t^{2} \kappa^{2}}{4 \epsilon(\nu+h)}\left\|\nabla\left(\frac{\mathbf{t u}{ }^{n+1}-\mathbf{t u}}{\Delta t}\right)\right\|^{2}+\epsilon(\nu+h)\left\|\nabla \boldsymbol{\phi}^{n+1}\right\|^{2}+\frac{C_{P F}^{2}}{4 \epsilon(\nu+h)}\left\|\frac{\boldsymbol{\eta}^{n+1}-\boldsymbol{\eta}^{n}}{\Delta t}\right\|^{2} \\
+\epsilon(\nu+h)\left\|\nabla \boldsymbol{\phi}^{n+1}\right\|^{2}+\frac{(\nu+h)}{4 \epsilon}\left\|\nabla \boldsymbol{\eta}^{n+1}\right\|^{2}+\epsilon(\nu+h)\left\|\nabla \boldsymbol{\phi}^{n+1}\right\|^{2}+\frac{C_{T r a c e}^{4} \kappa^{2}}{4 \epsilon(\nu+h)}\left\|\nabla \boldsymbol{\eta}^{n+1}\right\|^{2}+\frac{\kappa}{2}\left\|\boldsymbol{\phi}_{1}^{n+1}\right\|_{I}^{2} \\
+\frac{\kappa}{2}\left\|\phi_{2}^{n+1}\right\|_{I}^{2}+\frac{\kappa}{2}\left\|\phi_{1}^{n}\right\|_{I}^{2}+\frac{\kappa}{2}\left\|\boldsymbol{\phi}_{2}^{n}\right\|_{I}^{2}+\epsilon(\nu+h)\left\|\nabla \boldsymbol{\phi}^{n+1}\right\|^{2}+\frac{C_{T r a c e}^{4} \kappa^{2}}{4 \epsilon(\nu+h)}\left\|\nabla \boldsymbol{\eta}^{n}\right\|^{2}+2 \epsilon(\nu+h)\left\|\nabla \boldsymbol{\phi}^{n+1}\right\|^{2} \\
+\frac{|b|^{2}}{4 \epsilon(\nu+h)}\left\|\boldsymbol{\eta}^{n+1}\right\|^{2}+\frac{|b|^{2}}{4 \epsilon(\nu+h)}\left\|\boldsymbol{\phi}^{n}\right\|^{2}
\end{gathered}
$$

Moving the four boundary integrals from the RHS to the LHS, choosing $\epsilon=\frac{1}{18}$, summing over the time levels, using the modified Gronwall's lemma (notice that the last term in the RHS contains the sum over time levels up to $n$ only) and the triangle inequality (to pass from $\phi^{n+1}$ to $\mathbf{e}^{n+1}$ ) completes the proof.

In order to prove the accuracy estimate for the correction approximation, we will need the following

\subsection{Accuracy of Time Derivative of the Error in the Defect Step}

Theorem 7. (Accuracy of Time Derivative of the Error in the Defect Step) Let the assumptions of Theorem 6 be satisfied. Also, let $\Delta \mathbf{u} \in L^{2}\left(0, T ; L^{2}(\Omega)\right)$ and $\mathbf{u}_{t t t} \in L^{2}\left(0, T ; L^{2}(\Omega)\right)$. Then $\exists C>0$ independent of $h, \Delta t$ such that for any $n \in\left\{0,1,2, \cdots, M-1=\frac{T}{\Delta t}-1\right\}$, the discrete time derivative of the error $\frac{e_{i}^{n+1}-e_{n}^{i}}{\Delta t}$ satisfies

$$
\left\|\frac{\mathbf{e}^{n+1}-\mathbf{e}^{n}}{\Delta t}\right\|^{2}+(\nu+h) \Delta t \sum_{j=1}^{n}\left\|\nabla\left(\frac{\mathbf{e}^{j+1}-\mathbf{e}^{j}}{\Delta t}\right)\right\|^{2}+\frac{\kappa \Delta t}{2} \sum_{j=1}^{n} \sum_{i=1}^{2}\left\|\frac{e_{i}^{j+1}-e_{i}^{j}}{\Delta t}\right\|_{I}^{2} \leq C\left(h^{2}+(\Delta t)^{2}\right) .
$$

Proof. Taking $v_{i}=\frac{\phi_{i}^{n+1}-\phi_{i}^{n}}{\Delta t} \in X_{i, h}$ in (7.3) leads to

$$
\begin{array}{r}
\left(\frac{e_{i}^{n+1}-e_{i}^{n}}{\Delta t}, \frac{\phi_{i}^{n+1}-\phi_{i}^{n}}{\Delta t}\right)_{\Omega_{i}}+\left(\nu_{i}+h\right)\left(\nabla e_{i}^{n+1}, \nabla\left(\frac{\phi_{i}^{n+1}-\phi_{i}^{n}}{\Delta t}\right)\right)_{\Omega_{i}} \\
+\kappa \int_{I}\left(e_{i}^{n+1}-e_{j}^{n}\right) \frac{\phi_{i}^{n+1}-\phi_{i}^{n}}{\Delta t} d s+\left(b_{i} \cdot \nabla e_{i}^{n}, \frac{\phi_{i}^{n+1}-\phi_{i}^{n}}{\Delta t}\right)_{\Omega_{i}} \\
=h\left(\nabla t u_{i}^{n+1}, \nabla\left(\frac{\phi_{i}^{n+1}-\phi_{i}^{n}}{\Delta t}\right)\right)_{\Omega_{i}}+\left(\rho_{i}^{n+1}, \frac{\phi_{i}^{n+1}-\phi_{i}^{n}}{\Delta t}\right)_{\Omega_{i}} \\
-\Delta t\left(b_{i} \cdot \nabla\left(\frac{t u_{i}^{n+1}-t u_{i}^{n}}{\Delta t}\right), \frac{\phi_{i}^{n+1}-\phi_{i}^{n}}{\Delta t}\right)+\Delta t \kappa \int_{I}\left(\frac{t u_{j}^{n+1}-t u_{j}^{n}}{\Delta t}\right) \frac{\phi_{i}^{n+1}-\phi_{i}^{n}}{\Delta t} d s, i \neq j .
\end{array}
$$


Also, take $v_{i}=\frac{\phi_{i}^{n+1}-\phi_{i}^{n}}{\Delta t}$ in (7.3) at the previous time level, and subtract the resulting equation from (7.7). Denoting $s_{i}^{n+1} \equiv \frac{\phi_{i}^{n+1}-\phi_{i}^{n}}{\Delta t}$, summing over $i=1,2$ we obtain for $n \geq 1$

$$
\begin{array}{r}
\left\|\mathbf{s}^{n+1}\right\|^{2}-\left(\mathbf{s}^{n+1}, \mathbf{s}^{n}\right)+(\nu+h) \Delta t\left\|\nabla \mathbf{s}^{n+1}\right\|^{2}+\Delta t\left(b \cdot \nabla \mathbf{s}^{n}, \mathbf{s}^{n+1}\right) \\
+\sum_{i, j=1,2, i \neq j} \Delta t \int_{I} \kappa\left(s_{i}^{n+1}-s_{j}^{n}\right) s_{i}^{n+1} d s=\Delta t\left(\frac{\boldsymbol{\eta}^{n+1}-2 \boldsymbol{\eta}^{n}+\boldsymbol{\eta}^{n-1}}{(\Delta t)^{2}}, \mathbf{s}^{n+1}\right) \\
+(\nu+h) \Delta t\left(\nabla\left(\frac{\boldsymbol{\eta}^{n+1}-\boldsymbol{\eta}^{n}}{\Delta t}\right), \nabla \mathbf{s}^{n+1}\right)+\Delta t\left(b \cdot \nabla\left(\frac{\boldsymbol{\eta}^{n}-\boldsymbol{\eta}^{n-1}}{\Delta t}\right), \mathbf{s}^{n+1}\right) \\
+\sum_{i, j=1,2, i \neq j} \Delta t \int_{I} \kappa\left(\frac{\eta_{i}^{n+1}-\eta_{i}^{n}}{\Delta t}-\frac{\eta_{j}^{n}-\eta_{j}^{n-1}}{\Delta t}\right) s_{i}^{n+1} d s+h \Delta t\left(\nabla\left(\frac{\mathbf{t u}^{n+1}-\mathbf{t u}^{n}}{\Delta t}\right), \nabla \mathbf{s}^{n+1}\right) \\
+\Delta t\left(\frac{\rho^{n+1}-\rho^{n}}{\Delta t}, \mathbf{s}^{n+1}\right)+\sum_{i, j=1,2, i \neq j}(\Delta t)^{2} \int_{I} \kappa\left(\frac{\mathbf{t u}_{j}^{n+1}-2 \mathbf{t} \mathbf{u}_{j}^{n}+\mathbf{t u}_{j}^{n-1}}{(\Delta t)^{2}}\right) \mathbf{s}^{n+1} d s
\end{array}
$$

Using the Cauchy-Schwarz and Young's inequalities leads to

$$
\begin{array}{r}
\frac{1}{2}\left\|\mathbf{s}^{n+1}\right\|^{2}-\frac{1}{2}\left\|\mathbf{s}^{n}\right\|^{2}+(\nu+h) \Delta t\left\|\nabla \mathbf{s}^{n+1}\right\|^{2}+\frac{\Delta t \kappa}{2}\left\|s_{1}^{n+1}\right\|_{I}^{2}+\frac{\Delta t \kappa}{2}\left\|s_{2}^{n+1}\right\|_{I}^{2} \\
\leq \epsilon(\nu+h) \Delta t\left\|\nabla \mathbf{s}^{n+1}\right\|^{2}+\frac{C_{P F}^{2} \Delta t}{4 \epsilon(\nu+h)}\left\|\frac{\boldsymbol{\eta}^{n+1}-2 \boldsymbol{\eta}^{n}+\boldsymbol{\eta}^{n-1}}{(\Delta t)^{2}}\right\|^{2} \\
+\epsilon(\nu+h) \Delta t\left\|\nabla \mathbf{s}^{n+1}\right\|^{2}+\frac{1}{4 \epsilon(\nu+h)} \Delta t\|b\|^{2}\left\|\mathbf{s}^{n}\right\|^{2} \\
+\epsilon(\nu+h) \Delta t\left\|\nabla \mathbf{s}^{n+1}\right\|^{2}+\frac{(\nu+h) \Delta t}{4 \epsilon}\left\|\nabla\left(\frac{\boldsymbol{\eta}^{n+1}-\boldsymbol{\eta}^{n}}{\Delta t}\right)\right\|^{2} \\
+\epsilon(\nu+h) \Delta t\left\|\nabla \mathbf{s}^{n+1}\right\|^{2}+\frac{\|b\|^{2} \Delta t}{4 \epsilon(\nu+h)}\left\|\frac{\boldsymbol{\eta}^{n+1}-\boldsymbol{\eta}^{n}}{\Delta t}\right\|^{2}+2 \epsilon(\nu+h) \Delta t\left\|\nabla \mathbf{s}^{n+1}\right\|^{2} \\
+\frac{C_{T r a c e}^{4} \kappa \Delta t}{4 \epsilon(\nu+h)}\left(\left\|\nabla\left(\frac{\boldsymbol{\eta}^{n+1}-\boldsymbol{\eta}^{n}}{\Delta t}\right)\right\|^{2}+\left\|\nabla\left(\frac{\boldsymbol{\eta}^{n}-\boldsymbol{\eta}^{n-1}}{\Delta t}\right)\right\|^{2}\right)+\epsilon(\nu+h) \Delta t\left\|\nabla \mathbf{s}^{n+1}\right\|^{2} \\
+\frac{h^{2} \Delta t}{4 \epsilon(\nu+h)}\left\|\nabla\left(\frac{\mathbf{t u} \mathbf{u}^{n+1}-\mathbf{t u}}{\Delta t}\right)\right\|^{2}+\epsilon(\nu+h) \Delta t\left\|\nabla \mathbf{s}^{n+1}\right\|^{2} \\
+\frac{C_{P F}^{2} \Delta t}{4 \epsilon(\nu+h)}\left\|\frac{\rho^{n+1}-\rho^{n}}{\Delta t}\right\|^{2}+\frac{\Delta t \kappa}{4}\left\|s_{1}^{n+1}\right\|_{I}^{2} \\
+\frac{\Delta t \kappa}{4}\left\|s_{2}^{n+1}\right\|_{I}^{2}+(\Delta t)^{2} \Delta t \kappa \sum_{i=1,2}\left\|\frac{t u_{i}^{n+1}-2 t u_{i}^{n}+t u_{i}^{n-1}}{(\Delta t)^{2}}\right\|_{I}^{2} .
\end{array}
$$

Summing over the time levels, multiplying both sides by 2 , letting $\epsilon=\frac{1}{16}$ and using the modified Gronwall's lemma gives

$$
\left\|\mathbf{s}^{n+1}\right\|^{2}+(\nu+h) \Delta t \sum_{i=2}^{n+1}\left\|\nabla \mathbf{s}^{i}\right\|^{2}+\frac{\kappa \Delta t}{2} \sum_{i=2}^{n+1} \sum_{j=1}^{2}\left\|s_{j}^{i}\right\|_{I}^{2} \leq C\left(\left\|\mathbf{s}^{1}\right\|^{2}+O\left(h^{2}+(\Delta t)^{2}\right)\right)
$$

In order to get a bound on $\left\|\mathbf{s}^{1}\right\|^{2}$, consider (7.3) at $n=0$. Note also that we choose $u_{i}^{0}$ so that $\left(t u_{i}^{0}-u_{i}^{0}, v_{i}\right)=0, \forall v_{i} \in X_{i}^{h}, i=1,2$. Thus, $\mathbf{e}^{\mathbf{0}}=-\boldsymbol{\eta}^{\mathbf{0}}$ and $\boldsymbol{\phi}^{0}=0$. We let $\mathbf{v}=\mathbf{s}^{1}=\frac{\phi^{1}-\phi^{0}}{\Delta t}=\frac{\phi^{1}}{\Delta t}$ 
to obtain

$$
\begin{aligned}
& \left\|\mathbf{s}^{1}\right\|^{2}+\frac{(\nu+h)}{\Delta t}\left\|\nabla \boldsymbol{\phi}^{1}\right\|^{2}+\kappa\left\|\phi_{1}^{1}\right\|_{I}^{2}+\kappa\left\|\phi_{2}^{1}\right\|_{I}^{2}=\left(\frac{\boldsymbol{\eta}^{1}-\boldsymbol{\eta}^{0}}{\Delta t}, \mathbf{s}^{1}\right)+(\nu+h)\left(\nabla \boldsymbol{\eta}^{1}, \nabla \mathbf{s}^{1}\right)+\left(b \cdot \nabla \boldsymbol{\eta}^{0}, \mathbf{s}^{1}\right) \\
+ & \sum_{i, j=1,2, i \neq j} \int_{I} \kappa\left(\eta_{i}^{1}-\eta_{j}^{0}\right) s_{i}^{1} d s+h\left(\nabla \mathbf{t} \mathbf{u}^{1}, \nabla \mathbf{s}^{1}\right)+\left(\rho^{1}, \mathbf{s}^{1}\right)+\sum_{i, j=1,2, i \neq j} \Delta t \int_{I} \kappa\left(\frac{t u_{j}^{1}-t u_{j}^{0}}{\Delta t}\right) s_{i}^{1} d s
\end{aligned}
$$

Using the Cauchy-Schwarz and Young's inequalities, we show the following

$$
\begin{gathered}
\left\|\mathbf{s}^{1}\right\|^{2}+\frac{(\nu+h)}{\Delta t}\left\|\nabla \boldsymbol{\phi}^{1}\right\|^{2}+\frac{\kappa}{2}\left\|\phi_{1}^{1}\right\|_{I}^{2}+\frac{\kappa}{2}\left\|\boldsymbol{\phi}_{2}^{1}\right\|_{I}^{2} \\
\leq C\left[\left\|\frac{\boldsymbol{\eta}^{1}-\boldsymbol{\eta}^{0}}{\Delta t}\right\|^{2}+(\nu+h)\left\|\Delta \boldsymbol{\eta}^{1}\right\|^{2}+\|b\|^{2}\left\|\nabla \boldsymbol{\eta}^{0}\right\|^{2}\right. \\
\left.+\sum_{i=0,1, j=1,2} \kappa\left\|\nabla \eta_{j}^{i}\right\|^{2}+h^{2}\left\|\Delta \mathbf{t} \mathbf{u}^{1}\right\|^{2}+\left\|\rho^{1}\right\|^{2}+(\Delta t)^{2} \kappa \sum_{j=1,2}\left\|\frac{t u_{j}^{1}-t u_{j}^{0}}{\Delta t}\right\|_{I}^{2}\right]
\end{gathered}
$$

Inserting (7.12) into (7.10) completes the proof.

We now have all the intermediate results that are needed for proving the accuracy of the correction step solution $\mathbf{c u}$.

\subsection{Accuracy of Correction Step}

Theorem 8. (Accuracy of Correction Step) Let the assumptions of Theorem 7 be satisfied. Then $\exists C>0$ independent of $h, \Delta t$ such that for any $n \in\left\{0,1,2, \cdots, M-1=\frac{T}{\Delta t}-1\right\}$, the solution $\mathrm{cu}_{i}^{n+1}$ of (5.9) satisfies

$$
\left\|\mathbf{t} \mathbf{u}^{n+1}-\mathbf{c u}^{n+1}\right\|^{2}+(\nu+h) \Delta t \sum_{j=1}^{n+1}\left\|\nabla\left(\mathbf{t} \mathbf{u}^{j}-\mathbf{c u}^{j}\right)\right\|^{2}+\kappa \Delta t \sum_{i=1}^{2}\left\|t u_{i}^{n+1}-c u_{i}^{n+1}\right\|_{I}^{2} \leq C\left(h^{4}+(\Delta t)^{4}\right)
$$

Proof. First, sum (7.2) at time levels $t_{n}$ and $t_{n+1}$ and divide by 2 , to obtain in $\Omega_{i}, i=1,2$ :

$$
\begin{aligned}
&\left(\frac{t u_{i}^{n+1}-t u_{i}^{n}}{\Delta}, v_{i}\right)+\left(\nu_{i}+h\right)\left(\nabla t u_{i}^{n+1}, \nabla v_{i}\right)+\int_{I} \kappa\left(t u_{i}^{n+1}-t u_{j}^{n}\right) v_{i} d s+\left(b_{i} \cdot \nabla t u_{i}^{n}, v_{i}\right) \\
&=\left(\frac{f_{i}\left(t_{n+1}\right)+f_{i}\left(t_{n}\right)}{2}, v_{i}\right)+\frac{\Delta t\left(\nu_{i}+h\right)}{2}\left(\nabla\left(\frac{t u_{i}^{n+1}-t u_{i}^{n}}{\Delta t}\right), \nabla v_{i}\right)+h\left(\nabla\left(\frac{t u_{i}^{n+1}+t u_{i}^{n}}{2}\right), \nabla v_{i}\right) \\
&+ \frac{\kappa \Delta t}{2} \int_{I}\left(\frac{t u_{i}^{n+1}-t u_{i}^{n}}{\Delta t}\right) v_{i} d s+\frac{\kappa \Delta t}{2} \int_{I}\left(\frac{t u_{j}^{n+1}-t u_{j}^{n}}{\Delta t}\right) v_{i} d s-\frac{\Delta t}{2}\left(b_{i} \cdot \nabla\left(\frac{t u_{i}^{n+1}-t u_{i}^{n}}{\Delta t}\right), v_{i}\right) \\
&+\left(\frac{t u_{i}^{n+1}-t u_{i}^{n}}{\Delta t}-\frac{t u_{i, t}^{n+1}+t u_{i, t}^{n}}{2}, v_{i}\right)
\end{aligned}
$$

For the $O\left(\Delta t^{2}\right)$-term introduce the notation $\left(\frac{t u_{i}^{n+1}-t u_{i}^{n}}{\Delta t}-\frac{t u_{i, t}^{n+1}+t u_{i, t}^{n}}{2}, v_{i}\right) \equiv \gamma_{i}^{n+1}$. Subtract 
the correction step equation (5.9) from (7.14). We obtain for $i, j=1,2, i \neq j$

$$
\begin{gathered}
\left(\frac{c e_{i}^{n+1}-c e_{i}^{n}}{\Delta t}, v_{i}\right)+\left(\nu_{i}+h\right)\left(\nabla c e_{i}^{n+1}, \nabla v_{i}\right)+\int_{I} \kappa\left(c e_{i}^{n+1}-c e_{j}^{n}\right) v_{i} d s+\left(b \cdot \nabla c e_{i}^{n}, v_{i}\right) \\
=\frac{\Delta t\left(\nu_{i}+h\right)}{2}\left(\nabla\left(\frac{e_{i}^{n+1}-e_{i}^{n}}{\Delta t}\right), \nabla v_{i}\right)+h\left(\nabla\left(\frac{e_{i}^{n+1}+e_{i}^{n}}{2}\right), \nabla v_{i}\right)+\left(\gamma_{i}^{n+1}, v_{i}\right) \\
+\frac{\kappa \Delta t}{2} \int_{I}\left(\frac{e_{i}^{n+1}-e_{i}^{n}}{\Delta t}\right) v_{i} d s+\frac{\kappa \Delta t}{2} \int_{I}\left(\frac{e_{j}^{n+1}-e_{j}^{n}}{\Delta t}\right) v_{i} d s-\frac{\Delta t}{2}\left(b_{i} \cdot \nabla\left(\frac{e_{i}^{n+1}-e_{i}^{n}}{\Delta t}\right), v_{i}\right)
\end{gathered}
$$

Similarly to the error decomposition in the case of the defect approximation, decompose $c e_{i}^{n+1}=$ $t u_{i}^{n+1}-c u_{i}^{n+1}=\phi_{i}^{n+1}-\eta_{i}^{n+1}, \phi_{i} \in X_{i, h}$. We now choose $v_{i}=\phi_{i}^{n+1} \in X_{i, h}$ in (7.15), sum over $i=1,2$ and use the Cauchy-Schwarz and Young's inequalities to obtain bounds on the terms in (7.15), similar to what we did for equation (7.4). The bounds on $\mathbf{e}^{\mathbf{n}+\mathbf{1}}$ and $\frac{\mathbf{e}^{\mathbf{n}+\mathbf{1}}-\mathbf{e}^{\mathbf{n}}}{\Delta t}$ from Theorem 6 and Theorem 7 complete the proof. 


\section{Computational Testing}

The convergence properties of the two-step DDC method (Algorithm 5.1) are investigated quantitatively in the case of a test problem with the known solution (see [15]).

Assume $\Omega_{1}=[0,1] \times[0,1]$ and $\Omega_{2}=[0,1] \times[-1,0]$, so $I$ is the portion of the $x$-axis from 0 to 1 . Then $\mathbf{n}_{1}=[0,-1]^{T}$ and $\mathbf{n}_{2}=[0,1]^{T}$. For $\nu_{1}, \nu_{2}$, and $\kappa$ all arbitrary positive constants, the right hand side function $\mathbf{f}$ from (4.1) is calculated so that the true solution is given by $u_{i}=\left(u_{i 1}, u_{i 2}\right), i=1,2$

$$
\begin{aligned}
& u_{11}(t, x, y)=x(1-x)(1-y) e^{-t} \\
& u_{12}(t, x, y)=-x(1-x)(1-y) e^{-t} \\
& u_{21}(t, x, y)=x(1-x)\left(1+\frac{\nu_{1}}{\kappa}-\frac{\nu_{1}}{\nu_{2}} y-\left(1+\frac{\nu_{1}}{\nu_{2}}+\frac{\nu_{1}}{\kappa}\right) y^{2}\right) e^{-t} \\
& u_{22}(t, x, y)=-x(1-x)\left(1+\frac{\nu_{1}}{\kappa}-\frac{\nu_{1}}{\nu_{2}} y-\left(1+\frac{\nu_{1}}{\nu_{2}}+\frac{\nu_{1}}{\kappa}\right) y^{2}\right) e^{-t} .
\end{aligned}
$$

This choice of $\mathbf{u}$ satisfies the interface conditions (4.2) and the boundary conditions (4.4) with $g_{1}=g_{2}=0$. The computations were performed using finite element spaces consisting of continuous piecewise polynomials of degree 2 . The code was implemented using the software package FreeFEM++ [21].

\subsection{Convergence rate study}

Computational results are provided for $\kappa=1$ and for the moderate $\left(\nu_{1}=\nu_{2}=1\right)$ and small $\left(\nu_{1}=\nu_{2}=0.00001\right)$ values of the diffusion coefficients. In the following tables, the norm $\|\mathbf{u}\|$ is the discrete $L^{2}\left(0, T ; L^{2}(\Omega)\right)$ norm, given by

$$
\|\mathbf{u}\|=\left(\sum_{n=1}^{N} \Delta t\left|\mathbf{u}\left(t_{n}\right)\right|_{L^{2}\left(\Omega_{i}\right)}^{2}\right)^{1 / 2},
$$

and $|\mathbf{u}|_{H^{1}}$ is the discrete $L^{2}\left(0, T ; H^{1}(\Omega)\right)$ seminorm, given by

$$
|\mathbf{u}|_{H^{1}}=\left(\sum_{n=1}^{N} \Delta t\left|\nabla \mathbf{u}\left(t_{n}\right)\right|_{L^{2}(\Omega)}\right)^{1 / 2},
$$

where $N=T / \Delta t$. Tables ?? and 2 compare the cases of modified vs. non-modified jump condition in Algorithm 5.1 for $\nu_{i}=1$. Tables 3 and 4 perform the same comparison for the case of convection-dominated flows at $\nu_{i}=0.00001$. The errors are calculated in the norms (8.1) and (8.2). 
Table 1: Errors for computed approximations, $\boldsymbol{\nu}=1$, non-modified jump condition

\begin{tabular}{|rr|rr|rr|}
\hline DEFECT & SUBSTEP & & & \\
$h$ & $\Delta t$ & $\left\|\mathbf{u}\left(t_{n}\right)-\mathbf{u}^{n}\right\|_{L^{2}}$ & rate & $\left|\mathbf{u}\left(t_{n}\right)-\mathbf{u}^{n}\right|_{H^{1}}$ & rate \\
\hline $1 / 4$ & $1 / 4$ & $4.09893 \mathrm{e}-2$ & & $1.53394 \mathrm{e}-1$ & \\
$1 / 8$ & $1 / 8$ & $2.4502 \mathrm{e}-2$ & 0.74 & $8.99462 \mathrm{e}-2$ & 0.77 \\
$1 / 16$ & $1 / 16$ & $1.34949 \mathrm{e}-2$ & 0.86 & $4.91759 \mathrm{e}-2$ & 0.87 \\
$1 / 32$ & $1 / 32$ & $710272 \mathrm{e}-3$ & 0.92 & $2.57988 \mathrm{e}-2$ & 0.93 \\
$1 / 64$ & $1 / 64$ & $364762 \mathrm{e}-3$ & 0.96 & $1.32293 \mathrm{e}-2$ & 0.96 \\
\hline \hline CORRECTION & SUBSTEP & & & & \\
$h$ & $\Delta t$ & $\left\|\mathbf{u}\left(t_{n}\right)-\mathbf{c u}^{n}\right\|_{L^{2}}$ & rate & $\left|\mathbf{u}\left(t_{n}\right)-\mathbf{c u}^{n}\right|_{H} 1$ & rate \\
\hline $1 / 4$ & $1 / 4$ & $1.50131 \mathrm{e}-2$ & & $5.94415 \mathrm{e}-2$ & \\
$1 / 8$ & $1 / 8$ & $5.37793 \mathrm{e}-3$ & 1.48 & $2.0774 \mathrm{e}-2$ & 1.51 \\
$1 / 16$ & $1 / 16$ & $1.85568 \mathrm{e}-3$ & 1.53 & $7.34807 \mathrm{e}-3$ & 1.49 \\
$1 / 32$ & $1 / 32$ & $7.06498 \mathrm{e}-4$ & 1.39 & $2.9411 \mathrm{e}-3$ & 1.32 \\
$1 / 64$ & $1 / 64$ & $3.08825 \mathrm{e}-4$ & 1.19 & $1.34023 \mathrm{e}-3$ & 1.13 \\
\hline \hline
\end{tabular}

Table 2: Errors for computed approximations, $\boldsymbol{\nu}=1$, modified jump condition

\begin{tabular}{|rr|rr|rr|}
\hline DEFECT & SUBSTEP & & & & \\
$h$ & $\Delta t$ & $\left\|\mathbf{u}\left(t_{n}\right)-\mathbf{u}^{n}\right\|_{L^{2}}$ & rate & $\left|\mathbf{u}\left(t_{n}\right)-\mathbf{u}^{n}\right|_{H^{1}}$ & rate \\
\hline $1 / 4$ & $1 / 4$ & $4.10392 \mathrm{e}-2$ & & $1.5309 \mathrm{e}-1$ & \\
$1 / 8$ & $1 / 8$ & $2.42801 \mathrm{e}-2$ & 0.75 & $8.9456 \mathrm{e}-2$ & 0.77 \\
$1 / 16$ & $1 / 16$ & $1.33056 \mathrm{e}-2$ & 0.86 & $4.87916 \mathrm{e}-2$ & 0.87 \\
$1 / 32$ & $1 / 32$ & $6.98608 \mathrm{e}-3$ & 0.92 & $2.55628 \mathrm{e}-2$ & 0.93 \\
$1 / 64$ & $1 / 64$ & $358342 \mathrm{e}-3$ & 0.96 & $1.3099 \mathrm{e}-2$ & 0.96 \\
\hline \hline CORRECTION & $\mathbf{S U B S T E P}$ & & & & \\
$h$ & $\Delta t$ & $\left\|\mathbf{u}\left(t_{n}\right)-\mathbf{c u}^{n}\right\|_{L^{2}}$ & rate & $\left|\mathbf{u}\left(t_{n}\right)-\mathbf{c u}^{n}\right|_{H^{1}}$ & rate \\
\hline $1 / 4$ & $1 / 4$ & $1.36326 \mathrm{e}-2$ & & $5.50424 \mathrm{e}-2$ & \\
$1 / 8$ & $1 / 8$ & $4.61153 \mathrm{e}-3$ & 1.56 & $1.80651 \mathrm{e}-2$ & 1.60 \\
$1 / 16$ & $1 / 16$ & $1.36977 \mathrm{e}-3$ & 1.75 & $5.36588 \mathrm{e}-3$ & 1.75 \\
$1 / 32$ & $1 / 32$ & $3.78436 \mathrm{e}-4$ & 1.85 & $1.49775 \mathrm{e}-3$ & 1.84 \\
$1 / 64$ & $1 / 64$ & $1.00268 \mathrm{e}-4$ & 1.91 & $4.02327 \mathrm{e}-4$ & 1.89 \\
\hline \hline
\end{tabular}


Table 3: Errors for computed approximations, $\boldsymbol{\nu}=10^{-5}$, non-modified jump condition

\begin{tabular}{|rr|rr|rr|}
\hline DEFECT & SUBSTEP & & & & \\
$h$ & $\Delta t$ & $\left\|\mathbf{u}\left(t_{n}\right)-\mathbf{u}^{n}\right\|_{L^{2}}$ & rate & $\left|\mathbf{u}\left(t_{n}\right)-\mathbf{u}^{n}\right|_{H^{1}}$ & rate \\
\hline $1 / 4$ & $1 / 4$ & $7.91115 \mathrm{e}-2$ & & $5.3402 \mathrm{e}-1$ & \\
$1 / 8$ & $1 / 8$ & $7.08314 \mathrm{e}-2$ & 0.15 & $5.44157 \mathrm{e}-1$ & -0.02 \\
$1 / 16$ & $1 / 16$ & $5.9474 \mathrm{e}-2$ & 0.25 & $5.6404 \mathrm{e}-1$ & -0.05 \\
$1 / 32$ & $1 / 32$ & $5.31807 \mathrm{e}-2$ & 0.16 & $6.32873 \mathrm{e}-1$ & -0.16 \\
$1 / 64$ & $1 / 64$ & $5.11973 \mathrm{e}-2$ & 0.05 & $7.7229 \mathrm{e}-1$ & -0.28 \\
\hline \hline CORRECTION & SUBSTEP & & & & \\
$h$ & $\Delta t$ & $\left\|\mathbf{u}\left(t_{n}\right)-\mathbf{c u}^{n}\right\|_{L^{2}}$ & rate & $\left|\mathbf{u}\left(t_{n}\right)-\mathbf{c u}^{n}\right|_{H}$ & rate \\
\hline $1 / 4$ & $1 / 4$ & $7.3621 \mathrm{e}-2$ & & $5.61272 \mathrm{e}-1$ & \\
$1 / 8$ & $1 / 8$ & $6.06277 \mathrm{e}-2$ & 0.28 & $5.98215 \mathrm{e}-1$ & -0.09 \\
$1 / 16$ & $1 / 16$ & $5.45552 \mathrm{e}-2$ & 0.15 & $6.79845 \mathrm{e}-1$ & -0.18 \\
$1 / 32$ & $1 / 32$ & $5.32717 \mathrm{e}-2$ & 0.03 & $8.45552 \mathrm{e}-1$ & -0.31 \\
$1 / 64$ & $1 / 64$ & $5.23807 \mathrm{e}-2$ & 0.02 & 1.10753 & -0.38 \\
\hline \hline
\end{tabular}

Table 4: Errors for computed approximations, $\boldsymbol{\nu}=10^{-5}$, modified jump condition

\begin{tabular}{|c|c|c|c|c|c|}
\hline DEFECT & SUBSTEP & & & & \\
\hline$h$ & $\Delta t$ & $\left\|\mathbf{u}\left(t_{n}\right)-\mathbf{u}^{n}\right\|_{L^{2}}$ & rate & $\left|\mathbf{u}\left(t_{n}\right)-\mathbf{u}^{n}\right|_{H^{1}}$ & rate \\
\hline $1 / 4$ & $1 / 4$ & $9.23371 \mathrm{e}-2$ & & $3.64616 \mathrm{e}-1$ & \\
\hline $1 / 8$ & $1 / 8$ & $7.30489 \mathrm{e}-2$ & 0.33 & $3.06128 \mathrm{e}-1$ & 0.25 \\
\hline $1 / 16$ & $1 / 16$ & $4.72726 \mathrm{e}-2$ & 0.62 & $2.27403 \mathrm{e}-1$ & 0.42 \\
\hline $1 / 32$ & $1 / 32$ & $2.71885 \mathrm{e}-2$ & 0.79 & $1.62003 \mathrm{e}-1$ & 0.48 \\
\hline $1 / 64$ & $1 / 64$ & $1.46477 \mathrm{e}-2$ & 0.89 & $1.14559 \mathrm{e}-1$ & 0.49 \\
\hline CORRECTION & SUBSTEP & & & & \\
\hline$h$ & $\Delta t$ & $\left\|\mathbf{u}\left(t_{n}\right)-\mathbf{c u}^{n}\right\|_{L^{2}}$ & rate & $\left|\mathbf{u}\left(t_{n}\right)-\mathbf{c u}^{n}\right|_{H^{1}}$ & rate \\
\hline $1 / 4$ & $1 / 4$ & $6.75345 \mathrm{e}-2$ & & $2.89442 \mathrm{e}-1$ & \\
\hline $1 / 8$ & $1 / 8$ & $3.59619 \mathrm{e}-2$ & 0.90 & $2.15663 \mathrm{e}-1$ & 0.42 \\
\hline $1 / 16$ & $1 / 16$ & $1.47159 \mathrm{e}-2$ & 1.28 & $1.55311 \mathrm{e}-1$ & 0.47 \\
\hline $1 / 32$ & $1 / 32$ & $5.43386 \mathrm{e}-3$ & 1.44 & $1.12958 \mathrm{e}-1$ & 0.45 \\
\hline $1 / 64$ & $1 / 64$ & $1.87323 \mathrm{e}-3$ & 1.54 & $8.05629 \mathrm{e}-2$ & 0.48 \\
\hline
\end{tabular}




\section{CONCLUSION}

Clearly, the correct way of implementing the proposed method is to modify the interface condition so that the diffusion coefficient is treated consistently throughout the problem. Also, when the convection-to-diffusion ratio is moderate $\left(\nu_{i}=1\right)$ the observed convergence rates are in full agreement with the theoretical findings. When the diffusion coefficient is very small compared to the spatial mesh diameter, convergence rates in the $L^{2}\left(0, T ; L^{2}(\Omega)\right)$-norm start to deteriorate, but the correction step still gives a clear advantage over the first order accurate defect approximation. However, the accuracy in the $L^{2}\left(0, T ; H^{1}(\Omega)\right)$-seminorm has decayed drastically; this is due to the fact that for the chosen values of $\nu$ the mesh is much too coarse; the term $\nu+h$, appearing in the error estimates of the method, is now almost equal to $h$, which immediately affects both the a priori error estimates and the computational results. This suggests, that if the legacy codes are to be used for the coupled convection-dominated convection-diffusion problem in the manner of Algorithm 5.1, then one has to refine the mesh substantially in order to capture the gradient of the true solution. Notice, however, that the solution itself is well modelled (in the $L^{2}\left(0, T ; L^{2}(\Omega)\right)$-norm) even on a coarse mesh. 


\section{REFERENCES}

[1] Adams, R.A., and Fournier, J.J.F., 2003, Sobolev Spaces, vol. 140 of Pure and Applied Mathematics, Academic Press.

[2] Aggul, M., Labovsky, A., 2016, A High Accuracy Minimally Invasive Regularization Technique for Navier-Stokes Equations at High Reynolds Number, submitted to Num. Methods for PDEs.

[3] Ascher, U. M., Ruuth, S. J., and Wetton, B. T. R., 1995, Implicit-explicit methods for time-dependent partial differential equations, SIAM J. Num. Anal. 32(3).

[4] Axelsson, O., And Layton,W., 1988, Optimal interior estimates for the defectcorrection, finite element approach to 2-D convection-diffusion problems, ICMA report 88-116, Univ. of Pittsburgh.

[5] Bernardi, C., Gomez, T. Chacon-Rebello M., Lewandowski, R., Murat, F., 2002, A model of two coupled turbulent fluids, Part II: Numerical approximations by spectral discretization, SIAM Jour. Num. Analysis, Vol. 40, No. 6, pp. 2368-2394.

[6] Blum, H., Lisky, S., and Rannacher, R., 1992, A Domain Splitting Algorithm for Parabolic Problems, Computing 49, 11-23.

[7] Bourlioux, A., Layton, A. T., Minion, M. L., 2003, High-Order Multi-Implicit Spectral Deferred Correction Methods for Problems of Reactive Flows, Journal of Computational Physics, Vol. 189, No. 2, pp. 651-675.

[8] Böhmer, K., Hemker, P. W., Stetter, H. J., 1984, The defect correction approach, in: K. Böhmer, H. J. Stetter (Eds.),Defect Correction Methods. Theory and Applications, Springer Verlag, pp. 1-32.

[9] Brenner, S.C., and Scott, L.R., 2002, The Mathematical Theory of Finite Element Methods, Springer-Verlag.

[10] Bresch, D., and Koko, J., 2006, Operator-Splitting and Lagrange Multiplier Domain Decomposition Methods for Numerical Simulation of Two Coupled Navier-Stokes Fluids, Int. J. Appl. Math. Comput. Sci., Vol. 16, No. 4, pp. 419-429.

[11] Burman, E., Fernández, Miguel A., 2009, Stabilization of explicit coupling in fluidstructure interaction involving fluid incompressibility, Comput. Methods Appl. Mech. Engrg. 198 , pp. 766-784. 
[12] Burman, E., Hansbo, P., 2009, Interior penalty stabilized Lagrange multiplier for the finite element solution of elliptic interface problems, to appear in IMA Journal of Numerical Analysis, available online April 7.

[13] Causin, P., Gerbeau, J.-F., and Nobile, F., 2005, Added-mass effect in the design of partitioned algorithms for fluid-structure problems, Comput. Meth. Appl. Mech. Engrg., Vol. 194, No. 42-44, pp. 4506-4527.

[14] Connors, J., Howell, J., and Layton, W., 2012, Decoupled time stepping methods for fluidfluid interaction, SIAM Jour. Num. Analysis, Vol. 50, No. 3, pp. 1297-1319.

[15] Connors, J., Howell, J., and Layton, W., 2009, Partitioned timestepping for a parabolic two domain problem, SIAM Jour. Num. Anal., Vol. 47, No. 5.

[16] Dawson, C. N., and Du, Q., 1991, A Finite Element Domain Decomposition Method for Parabolic Equations, Fourth International Symposium on Domain Decomposition Methods for PDEs, edited by R. Glowinski et al, pp. 255-263, SIAM, Philadelphia.

[17] Dutt, A., Greengard, L., Rokhlin, V., 2000, Spectral Deferred Correction Methods for Ordinary Differential Equations, BIT 40 (2) pp. 241-266.

[18] Ervin, V. J., Lee, H. K., 2006, Defect correction method for viscoelastic fluid flows at high Weissenberg number, Numerical Methods for Partial Differential Equations, Volume 22, Issue 1, pp. 145 - 164.

[19] Frank, R., Ueberhuber, W., 1977, Iterated Defect Correction for the Efficient Solution of Stiff Systems of Ordinary Differential Equations, BIT 17, pp. 146-159.

[20] Gunzburger, M., Labovsky, A., 2012, High Accuracy Method for Turbulent Flow Problems, M3AS: Mathematical Models and Methods in Applied Sciences, vol. 22 (6).

[21] Hecht, F., LeHyaric, A., and Pironneau, O., 2008. Freefem++ version 2.24-1. http://www.freefem.org/ff++.

[22] Heywood, J. G., and Rannacher, R., 1990, Finite-elements approximation of the nonstationary Navier-Stokes problem part IV: Error analysis for second-order discretization. SIAM J. Numer. Anal. , vol. 27(2), pp. 353-384.

[23] Labovsky, A., 2009, A Defect Correction Method for the Evolutionary Convection Diffusion Problem with Increased Time Accuracy, Computational Methods in Applied Mathematics, Vol. 9, No. 2, pp. 154-164. 
[24] Labovsky, A., 2008, A Defect Correction Method for the Time-Dependent Navier-Stokes Equations, Numerical Methods for Partial Differential Equations, vol.25(1), pp.1-25.

[25] Layton, W., Lee H. K., Peterson, J., 2002, A defect-correction method for the incompressible Navier-Stokes equations, Applied Mathematics and Computation, Vol. 129, Issue 1, pp. 1-19.

[26] Lewandowski, R., 1997, Analyse Mathématique et Océanographic, collection RMA, Masson

[27] Minion, M. L., 2003, Semi-Implicit Spectral Deferred Correction Methods for Ordinary Differential Equations, Comm. Math. Sci. 1, 471-500.

[28] Minion, M. L., 2004, Semi-Implicit Projection Methods for Incompressible Flow based on Spectral Deferred Corrections, Appl. Numer. Math., 48(3-4), 369-387.

[29] Minion, M. L., 2003, Semi-Implicit Projection Methods for Ordinary Differential Equations, Comm. Math. Sci., 1(3), 471-500.

[30] Stetter, H. J., 1978, The defect correction principle and discretization methods, $\mathrm{Nu-}$ merische Mathematik, vol. 29(4), pp. 425-443. 